\title{
Minería de datos distribuida usando clustering k-means en la predictibilidad del proceso petitorio en una organización pública
}

ZORAIDA MAMANi RODRÍGUEZ ${ }^{1}$ Luz Del Pino RodrígueZ ${ }^{2}$ Augusto CorteZ VASQueZ ${ }^{3}$

\section{RESUMEN}

La minería de datos distribuida está contemplada en el campo de la investigación e implica la aplicación del proceso de extracción de conocimiento sobre grandes volúmenes de información almacenados en bases de datos distribuidas. Las organizaciones modernas requieren de herramientas que realicen tareas de predicción, pronósticos, clasificación entre otros y en línea, sobre sus bases de datos que se ubican en diferentes nodos interconectados a través de internet, de manera que les permita mejorar la calidad de sus servicios. El Clustering es una de las principales tecnicas de modelado de la mineria de datos la cual consiste en dividir la información en grupos diferentes, internamente los miembros de cada grupo son muy similares unos de otros y disimiles respecto a los miembos de los otros grupos. Los grupos 0 clusters resultantes permiten predecir patrones de comportamiento que pueden aportar en la toma de decisiones de las organizaciones. Es en este contexto que e presente trabajo elabora una propuesta de un prototipo de aplicación de minería de datos distribuida basado en la técnica k-means en la predictibilidad del proceso petitorio de una organizacion pública.

Palabras clave: Minería de Datos Distribuida, Algoritmo Clustering, K-means, petitorio

APPLICATION OF THE DISTRIBUTED DATA MINING USING CLUSTERING K-MEANS IN THE PREDICTABILITY OF THE REQUEST PROCESS OF A PUBLIC ORGANIZATION

\section{ABSTRACT}

Distributed data mining is contemplated in the field of research and involves the application of the process of extracting knowledge about large volumes of information stored in distributed databases. Modern organizations require tools that perform tasks of prediction, forecasting, classification and others, online, on their databases that are located in different nodes interconnected through the Internet, in a way that allows them to improve the quality of their services. Clustering is one of the main modeling techniques of data mining which consists of dividing the information into different groups, internally the members of each group are very similar to each other and dissimilar to the members of the other groups. The resulting clusters or clusters allow us to predict patterns of behavior that can contribute to organizational decision-making. It is in this context that the present work elaborates a proposal of a prototype of application of distributed data mining based on the k-means technique in the predictibilidad of the request process of a public organization.

Keywords: Distributed Data Mining, Clustering Algorithm, K-means, Petition

\section{INTRODUCCIÓN}

La minería de datos distribuida es una disciplina de alto interés de los investigadores debido a las limitaciones que ofrece la mineria de datos centralizada a las realidades organizacionales actuales. Las organizaciones competitivas deben mantenerse dispuestas al cambio, a la mejora continua de los servicios que brindan, respetando los estándares de la industria. Por ello están interesadas en utilizar herramientas informáticas que apoyen sus objetivos.

El clustering es una de las principales tecnicas de modelado de la minería de datos la cual consiste en dividir la información en grupos diferentes, internamente los miembros de cada grupo son muy similares unos de otros y disimiles respecto a los miembos de los otros grupos. Los grupos o clusters pueden ser usados para clasificar nuevos datos (Hurtado, 2005).

En Rekha y Sabu (2010) se define tres modelos de arquitectura de mineria de datos distribuida; el primero consiste en que cada nodo distribuido dispone de un componente de minería encargado de minar los datos en la base de datos local, obteniéndose de esta forma, un modelo de minería de datos parcial en cada uno de los nodos; posteriormente, estos modelos parciales se combinan para obtener el modelo de minería de datos global. Los otros dos modelos son similares pues ambas consideran implementar un modelo global de minería de datos en la parte superior del sistema distribuido que actúe sobre una vista integrada de las distintas bases de datos locales. La diferencia entre estos dos modelos radica en la forma en que se genera la vista integrada sobre la que actuaría la capa de minería de datos. La primera realiza consultas en cada base de datos distribuida de manera independiente, generando un modelo de datos integrado sobre el que operan los algoritmos de minería de datos; mientras que la segunda integra todas las bases de datos distribuidas y las consultas se realizan sobre esta vista integrada de datos.

El organismo judicial peruano ha sido considerado en el presente estudio por tratarse de un modelo de organización moderna cuyos objetivos se encuentran establecidos en la agenda estratégica (Mendoza, 2013); documento que a su vez se encuentra alineado al Plan de desarrollo Institucional al 2018, Plan Bicentenario y al Plan Nacional para la Reforma Integral de la Administración de Justicia.

1 Docente Asociada de la Facultad de Ingeniería de Sistemas e Informatica de la UNMSM. E-mail: zmamanir@unmsm.edu.pe ORCID:0000-0002-2590-8387

2 Docente Asociada de la Facultad de Ingeniería de Sistemas e Informatica de la UNMSM. E-mail: 1delpinor@unmsm.edu.pe ORCID: 0000-0002-2893-8047

3 Docente Principal de la Facultad de Ingeniería de Sistemas e Informatica de la UNMSM. E-mail: acortezv@unmsm.edu.pe ORCID: 0000-0002-3752-4321 
Esta agenda está enfocada en tres ejes: el eje ciudadano, el eje interno y el eje externo. Asimismo, señala como uno de los objetivos principales la predictibilidad de las decisiones judiciales a nivel del eje ciudadano. Además, a nivel del eje interno se precisa como objetivos primordiales la gestión de la calidad jurisdiccional, el uso de las tecnologías de información y justicia electrónica con monitoreo permanente, el uso y análisis de la información estadística y de los indicadores de producción y de calidad.

La reducción de los tiempos procesales permitirá la reducción de la carga procesal del sector judicial; este es otro de los objetivos de la institución; para lo cual se viene realizando estudios de los procesos judiciales que presentan mayor carga procesal. En la Tabla $N^{\circ} 1$ se expone cuatro de los diez procesos identificados y de todas las especialidades en seis sedes judiciales. Asimismo Breña (2008) realiza un analisis completo de la carga procesal como barrera para el acceso a la justicia.

Tabla 1: Procesos con mayor carga procesal

\begin{tabular}{|c|c|c|c|}
\hline $\mathbf{N}^{\circ}$ & Procesos & $\begin{array}{l}\text { Tiempo } \\
\text { de } \\
\text { demora } \\
\text { al } 80 \% \\
\text { (dias) }\end{array}$ & $\begin{array}{c}\text { Motivos de demora que afectan la } \\
\text { sentencia }\end{array}$ \\
\hline 1 & Alimentos & $100-1200$ & $\begin{array}{l}\text { 1.- Oficios a organismos donde labora el } \\
\text { demandado y a otras entidades } \\
\text { 2.- Escritos relativos a la solicitud y reformulacion } \\
\text { de liquidaciones } \\
\text { 3.- Solicitud de desarchivamiento } \\
\text { 4.- Notificaciones }\end{array}$ \\
\hline 2 & \begin{tabular}{|l} 
Violencia \\
Familiar
\end{tabular} & $300-600$ & $\begin{array}{l}\text { 1.- Oficios relativos a informes psicologicos } \\
\text { 2.- Notificaciones }\end{array}$ \\
\hline 3 & Desalojo & $600-900$ & $\begin{array}{l}\text { 1.- Escrito para impulsar el proceso } \\
\text { 2.- Notificaciones }\end{array}$ \\
\hline 4 & $\begin{array}{l}\text { s de dar } \\
\text { sumas de } \\
\text { dinero }\end{array}$ & $900-1200$ & $\begin{array}{l}\text { 1.- Incumplimiento de requerimiento del juzgado } \\
\text { 2.- Escritos para impulsar el proceso } \\
\text { 3.- Resoluciones para conocimiento de las partes }\end{array}$ \\
\hline
\end{tabular}

Fuente: Proyecto de Mejoramiento de los Servicios de Justicia, 2014

El proceso de negocio sobre el cual se centrará el presente estudio es el petitorio. Veramendi (2008) define el petitorio como lo que se pide sea reconocido o declarado en la sentencia a favor del demandante.

"El petitum es el elemento fundamental de la pretensión del actor en relación con la congruencia de la sentencia ya que ni su objeto inmediato ni mediato puede modificarse a lo largo del proceso ni en la resolución judicial. En pocas palabras, la sentencia debe inexcusablemente ser congruente con la petición." Ezquiaga, 2000: 53 y Sendra, 2007: 209-210 (citado en Veramendi, 2008).

Es en este contexto que se plantea la presente investigación la cual desarrolla un prototipo que aplica minería de datos distribuida sobre datos nomi- nales para determinar patrones de comportamiento en el petitorio de la carga procesal de los periodos 2008 al 2010 correspondiente a un organo jurisdiccional casatorio.

\section{SOLUCIÓN PROPUESTA}

La metodología de desarrollo que se aplica para la implementación del prototipo de de Minería de Datos Distribuida (MDD) consiste en cinco fases:
A. Diseño del Modelo Dimensional
B. Diseño del Algoritmo MDD
C. Arquitectura del Prototipo
D. Diseño e Implementación del Prototipo
E. Determinación de Resultados

\subsection{Diseño del Modelo Dimensional}

La arquitectura de datos según la normalización de sus dimensiones es un esquema copo de nieve según lo define Kimball, Reeves, Ross y Thornthwaite (2002). En la Tabla 2 se describe las dimensiones y hechos concernientes al proceso de negocio petitorio.

Tabla 2: Descripción de Dimensiones y Hechos

\begin{tabular}{|c|c|}
\hline Dimensión & Descripción \\
\hline D01 dim cuadernillo & $\begin{array}{l}\text { Contiene información concerniente al } \\
\text { expediente judicial }\end{array}$ \\
\hline D02 dim recurso & $\begin{array}{l}\text { Contiene información referente al recurso } \\
\text { formulado en el petitorio: Casación, } \\
\text { Apelación, Queja, etc. }\end{array}$ \\
\hline D03 dim especialidad & $\begin{array}{l}\text { La especialidad a la cual corresponde el } \\
\text { proceso: Civil, Constitucional, Penal, } \\
\text { Laboral, Familia, entre otros. }\end{array}$ \\
\hline D04 dim materia & Clase a la que pertenece el proceso \\
\hline $\begin{array}{l}\text { D05 } \\
\text { dim_organo_jurisdiccional }\end{array}$ & $\begin{array}{l}\text { Corresponde a las Salas y juzgados que } \\
\text { integran el organismo judicial según la ley } \\
\text { orgánica del mismo, cada caso judicial } \\
\text { abierto está vinculado a un órgano } \\
\text { jurisdiccional. }\end{array}$ \\
\hline D06 dim tiempo & $\begin{array}{l}\text { Contiene información referente al calendarid } \\
\text { esta permite analizar la información } \\
\text { basándose en periodos de evaluación. }\end{array}$ \\
\hline D07 dim tema & $\begin{array}{l}\text { Contiene información referente al pedido } \\
\text { que realiza la parte procesal; no puede } \\
\text { cambiar durante el tiempo de vida del } \\
\text { proceso judicial. }\end{array}$ \\
\hline D08 dim norma & $\begin{array}{l}\text { Corresponde a la parte normativa, } \\
\text { fundamentos de ley, sobre la cual las partes } \\
\text { procesales fundamentan su pedido. }\end{array}$ \\
\hline F01 fact petitorio & $\begin{array}{l}\text { Contiene los hechos concerniente al proces } \\
\text { de negocio "Petitorio" }\end{array}$ \\
\hline
\end{tabular}

Fuente: Elaboración propia en base a Kimball, Reeves, Ross y Thornthwaite (2002) 


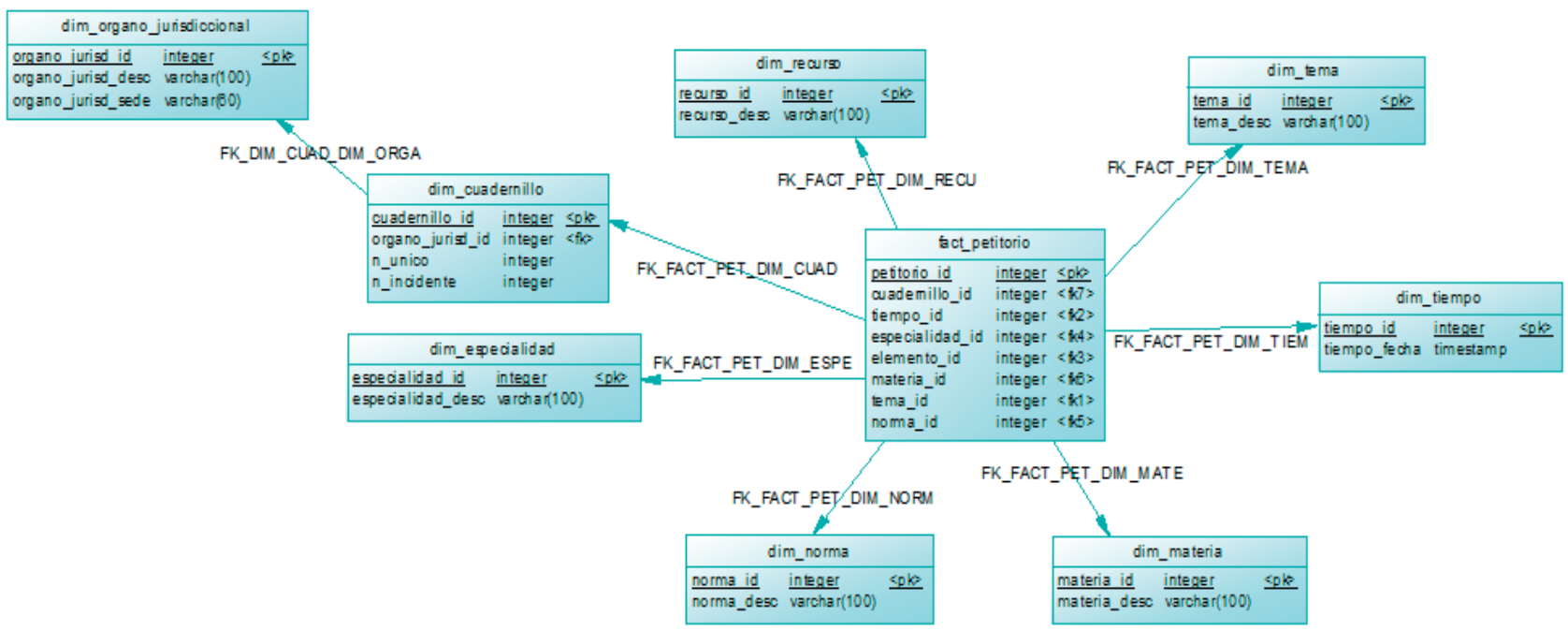

Figura 1: Modelo Dimensional Copo de Nieve

Fuente: Elaboracion Propia

En la figura 1 se presenta el diseño del modelo dimensional propuesto sobre el cual se circunscribe la aplicación y los resultados, la tabla fact_petitorio es la estructura que contendra a todos los hechos concernientes del proceso a evaluar, como se puede apreciar en la figura esta se interrelaciona con un conjunto de siete dimensiones; todo en conjunto permitirá formular el modelo de mineria de datos.

\subsection{Diseño del Algoritmo MDD}

Considerando el esquema distribuido que presenta el negocio en cuestión; una base de datos en cada sede judicial, se suma la sensibilidad y confidencialidad de la información que este tipo de organización maneja, infraestructura de tecnologías de información legadas; todo ello nos orienta al uso de minería de datos distribuida con lo cual se mantendrá la confidencialidad de la información al evitar su centralización, se reutilizará la infraestructura tecnológica y arquitectura de datos actual que presenta el negocio, se requiere mínimo presupuesto y se puede ver resultados a corto plazo.

La técnica de minería de datos elegida es K-means; esta técnica pertenece a la clase de técnicas de tipo clustering, esta permitirá agrupar los hechos del datamart propuesto según patrones de afinidad. El número de grupos o clusters resultantes ha obtener es configurable en nuestro caso definiremos cinco clusters inicialmente.

La técnica K-means presenta una serie de variantes de implementación; a continuación se formula una propuesta aplicable al contexto del presente estudio.

\subsection{Algoritmo}

La propuesta algorítmica consiste en el siguiente flujo:

1.- Sea $\Phi=\bigcup_{i=1}^{n} \beta_{i} \quad \forall i \varepsilon[1, n]$ el conjunto global de la información distribuida en sedes, donde cada $\beta_{i}$ representa la base de datos de la sede $i$ y ${ }^{n}$ representa el número de bases de datos de todas las sedes judiciales.

2.- Sea $\mu$ el modelo dimensional propuesto el cual se considera desplegado en las $n$ sedes.

3.- Se elige las sedes a incluir en la evaluación y se indicara el número de clusters a generar.

4.- Sea $\delta=\bigcup_{k=1}^{m} d_{k} \quad \forall k \varepsilon[1, m]$ el dataset resultante de la ejecución de $\mu$ en las sedes $m$ seleccionadas; considérese $m<=n$ respectivamente asimismo las instancias de $\delta$ son datos cualitativos y nominales.

5.- De $\delta$ elegir $c$ centroides aleatoriamente

6.- Determinar conglomerados considerando la distancia euclidiana de la instancia al centroide $s\left(\mathrm{Ii} \rightarrow \mathrm{C}_{\mathrm{s}}\right) \forall I_{i} \varepsilon \delta$; determinar la distancia de a $I_{i}$ cada centroide considerándose que la instancia $i$ pertenece al conglomerado si y solo si la distancia de la instancia al centroide es la mínima distancia determinada del conjunto de distancias calculadas para con respecto al conjunto de centroides.

$$
I_{i} \varepsilon C_{s} \therefore \operatorname{distancia}\left(I_{i}, C_{s}\right)=\min \left\{d_{i 1}, d_{i 2}, d_{i c}, \ldots d_{i(c-1)}, d_{i c}\right\}
$$


7.- Repetir el paso 5 y paso 6 hasta que los nuevos centroides determinados en la iteración sean los mismos de la iteración $p-1$ con lo cual se podría concluir el proceso iterativo.

8.- Presentar la distribución final:

$$
\begin{aligned}
& K_{1}=\bigcup_{i=1}^{a_{1}} I_{i} \quad \forall i \varepsilon\left[1, a_{1}\right], \quad K_{2}=\bigcup_{i=1}^{a_{2}} I_{i} \quad \forall i \varepsilon\left[1, a_{2}\right], \\
& K_{c}=\bigcup_{i=1}^{a_{m}} I_{i} \quad \forall i \varepsilon\left[1, a_{m}\right]
\end{aligned}
$$

Donde: $a_{1}+a_{2}+a_{3} \ldots+a_{m} \leq n, s \leq c$ y $K_{c}$

y son conjuntos de instancias disjuntos.

\subsection{Arquitectura del Prototipo}

La arquitectura del prototipo se enfoca en una arquitectura basada en tres capas, aplica el patrón arquitectonico Modelo Vista Controlador (MVC) Pressman (2010) en su diseño.

La figura 2 explica la arquitectura y su flujo se detalla a continuación:

El usuario inicia el proceso de clustering mediante el uso de un formulario web IU_Petitorio.jsp. Este evento es atendido por un Servlet: CTRL_Petitorio.

El servlet definido cumplirá el rol de controlador-delegador; este toma la petición procedente de la interfaz web y realiza las siguientes tareas:
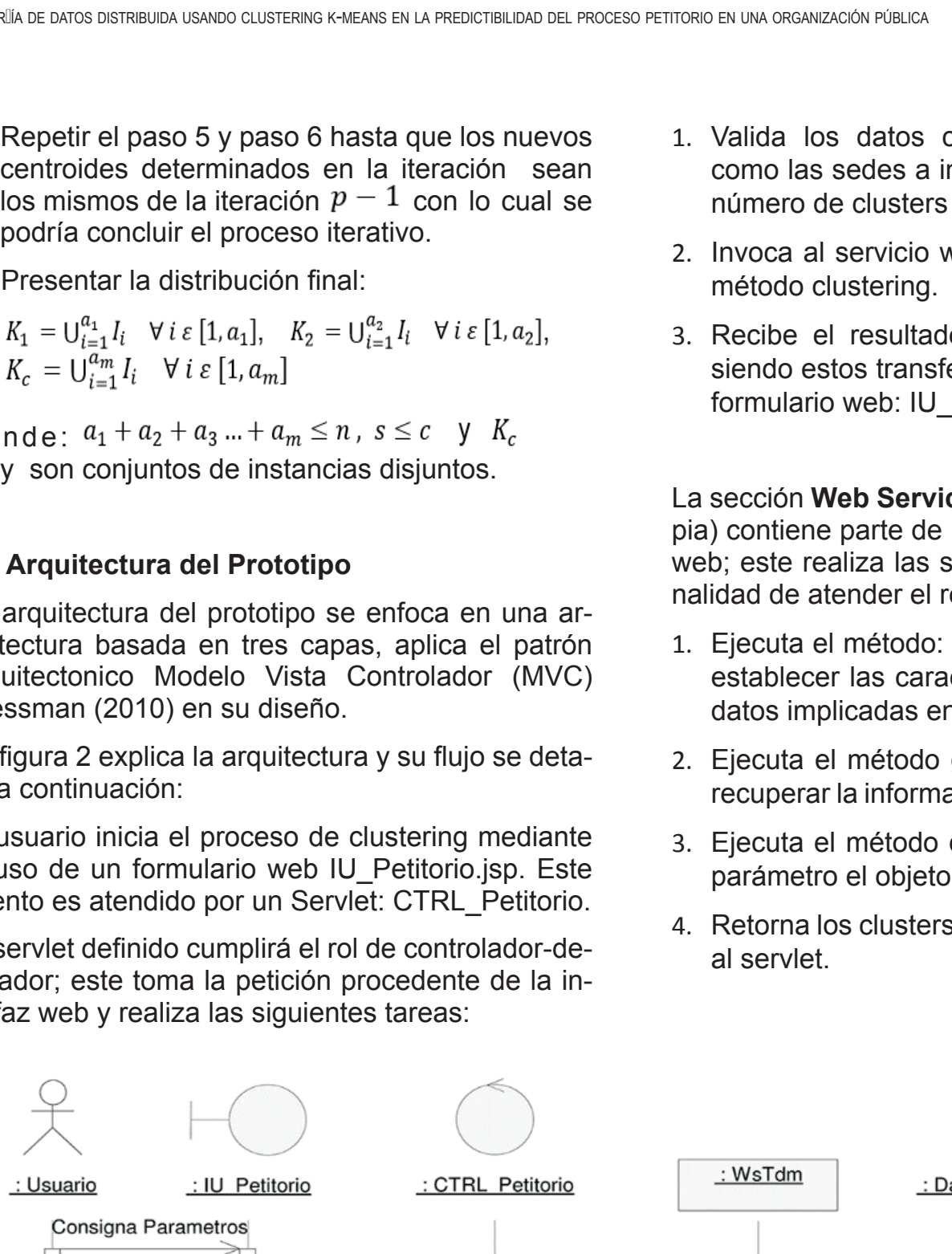

: Usuario

: IU Petitorio

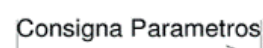

Consigna Parametros
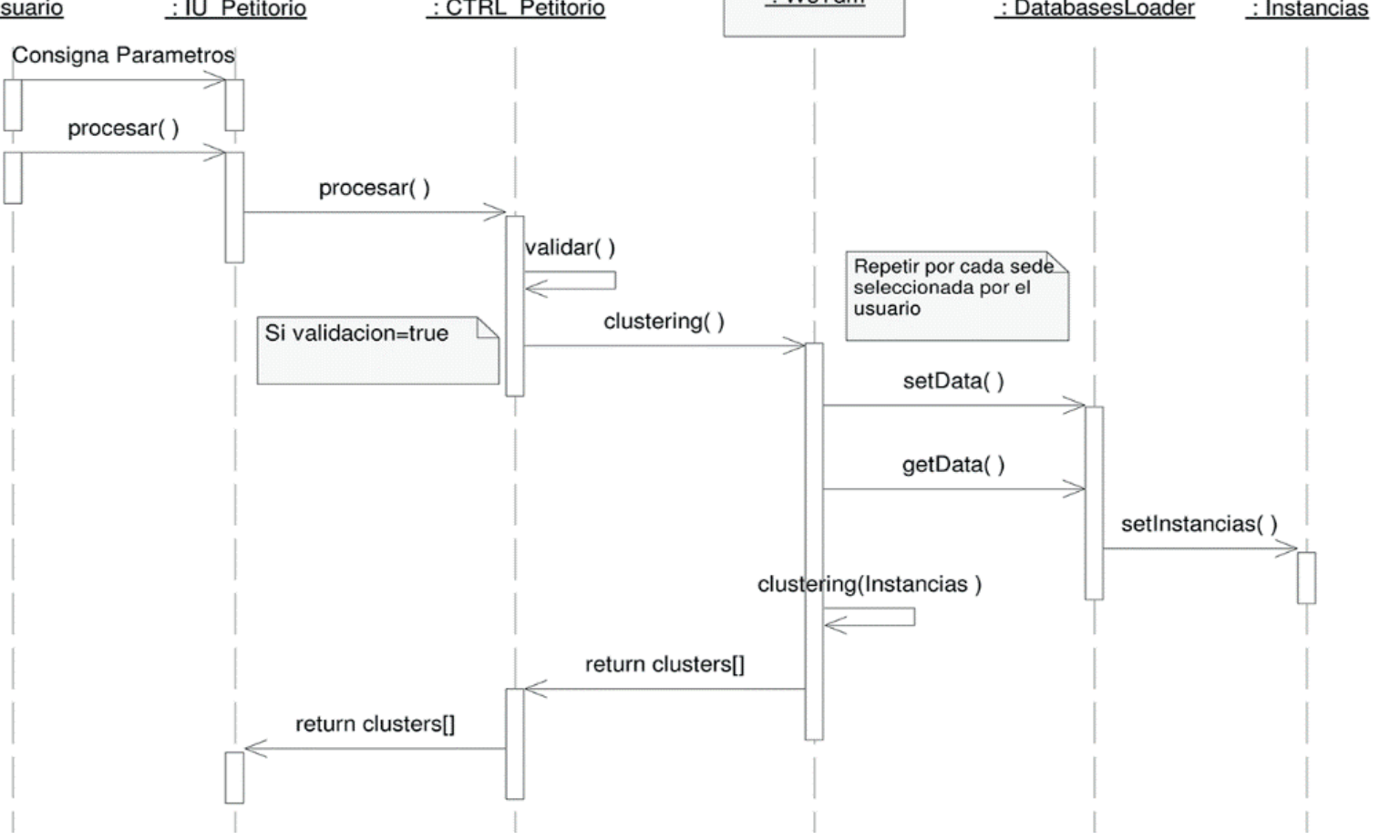

Figura 2: Arquitectura del Prototipo

Fuente: Elaboración propia 


\section{Web Service WsTdm}

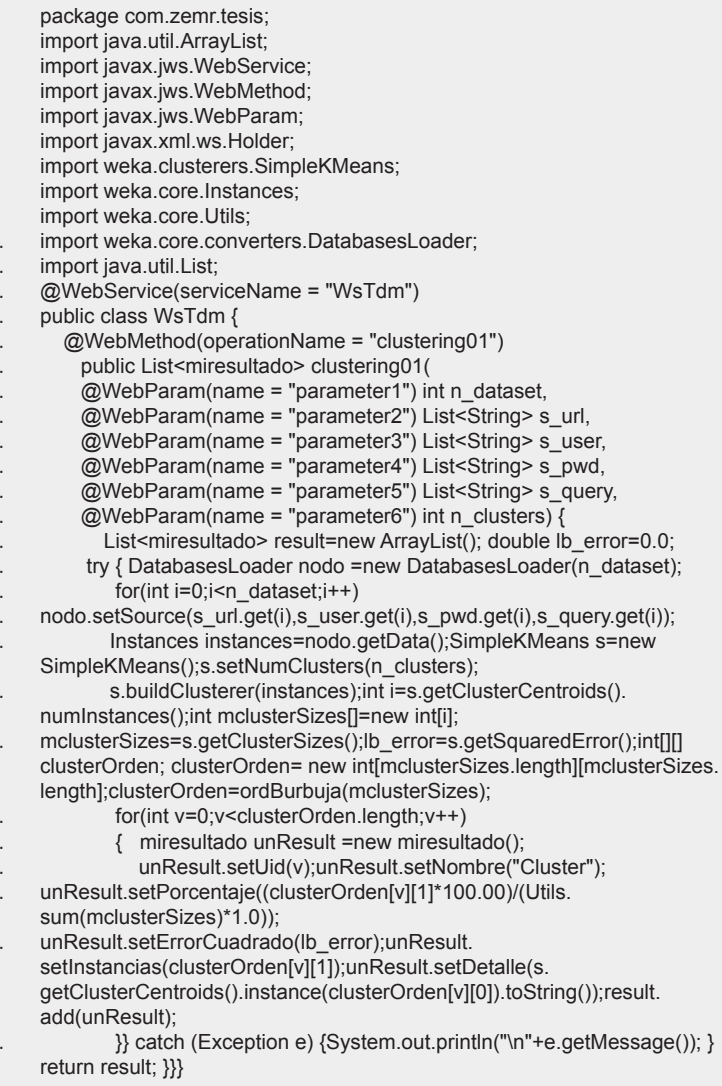

En Servlet Ctrl_Petitorio (Elaboración propia) se expone el código fuente del controlador, este objeto realiza la carga de las instancias de datos correspondiente a las $\mathrm{n}$ sedes definidas en la interfaz IU_Petitorio.jsp, asimismo es el encargado de invocar al servicio web, asignarle las instancias y recepcionar los resultados que este retorne convertirlo a formato JavaScript Object Notation (JSON) y remitirlo a la interfaz IU_Petitorio.jsp tal como se aprecia en la Figura 3.

\section{Servlet Ctrl_Petitorio}

package servlet

import com.zemr.tesis.WsTdm_Service;

import java.io.*;

import java.util.ArrayList;

import java.util.List;

import javax.servlet.ServletException;

import javax servlet http. HttpServlet;

import javax.serveth

import javax.servlet.http.HttpServletResponse;

import javax.servlet.RequestDispatcher;

import javax.xml.ws.WebServiceRe

import com.zemr.tesis.Miresultado;

import org.json.simple.*

public class CTRL_Petitorio extends HttpServlet \{

@WebServiceRef(wsdlLocation = "WEB-INF/wsdl/localhost 8080/

AppWsTdm/WsTdm.wsdl")

private WsTdm Service service;

@Override

@Override response)

throws ServletException, IOException \{

int $n \_$dataset $=0$, n_clusters $=0$, n_corte $=4$;

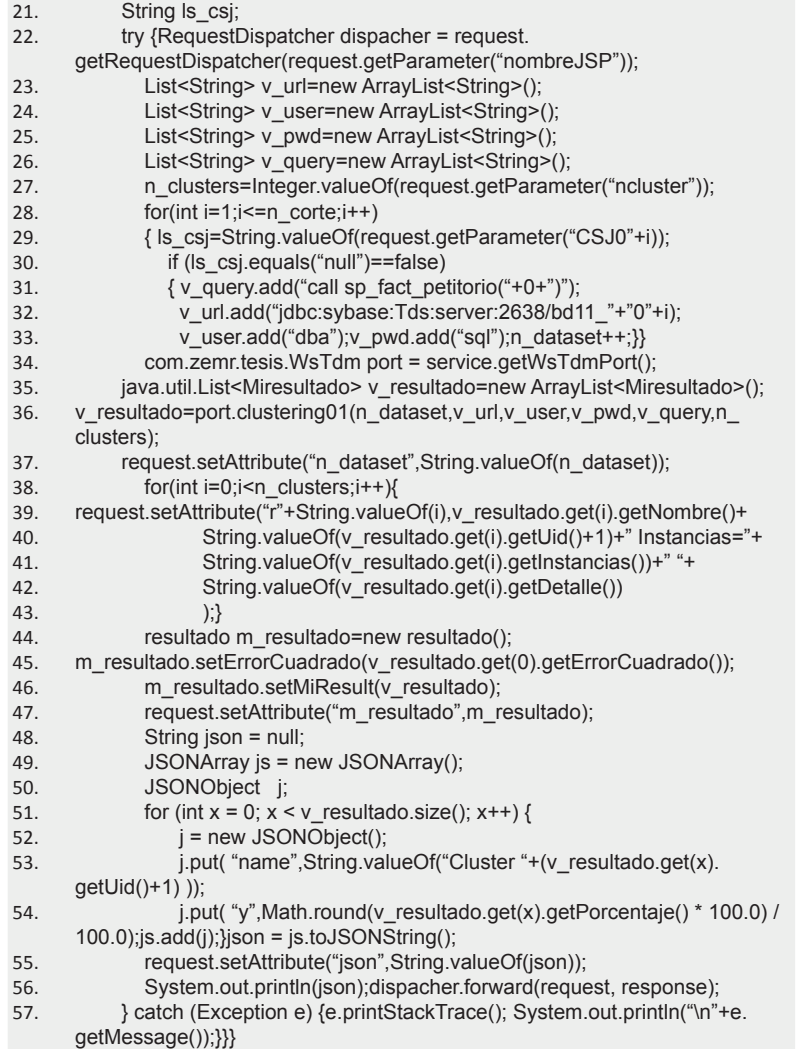

\subsection{Diseño del Prototipo}

El prototipo de aplicación de minería de datos propuesto es una aplicación web desarrollada en lenguaje de programación java según la arquitectura de aplicación definida en la Figura 2.

En la Figura 3 se expone el diseño del prototipo el cual se explica a continuación.

El formulario se divide en tres secciones: la primera sección presenta el título del proyecto. La segunda sección contiene la lista parcial de sedes judiciales consideradas en el presente proyecto y los botones Procesar y Limpiar. La tercera sección contiene los resultados de la evaluación del clustering; Se tiene un ítem por cada cluster o conglomerado generado.

La estructura del ítem consiste de: un número y/o identificador del cluster, el total de instancias que lo conforman, el porcentaje que representa este con respecto al total de las instancias evaluadas y por último el detalle del conglomerado; las características que definen el conglomerado o cluster resultante.

En la parte superior del formulario se puede apreciar el grafico estadístico que refleja los resultados de la evaluación en cantidades porcentuales.

La sección IU_Petitorio.jsp (Elaboración propia) corresponde al código fuente que sostiene al formulario web de la figura 3 , este código utiliza HTML 
5 para el diseño del formulario web, hojas de estilo, librerías javascript, jquery.js y highcharts.js para brindar la presentación que se ofrece en el formulario web y los gráficos estadísticos que esta contiene.

\section{IU_Petitorio.jsp}

<\%@page import="java.util.Iterator"\%>

<\%@page import="servlet.resultado"\%>

$<\% @$ page contentType="text/html" pageEncoding="UTF-8"\%> $<$ !DOCTYPE html>

$<\mathrm{html}>$

$<\%$ resultado $\mathrm{m}_{-}$resultado $=$(resultado)request.getAttribute $\left({ }^{\prime \prime} \mathrm{m}_{-}\right.$ resultado");

String $n \_$dataset $=$(String)request.getAttribute("n_dataset");

String result="'; \%>

$<$ head $>$

$<$ meta http-equiv="Content-Type" content="text/html; charset=UTF-8"> <title>Prototipo Aplicacion DDM</title>

<link rel="shortcut icon" href="itinnovacionLogo.jpg">

$<$ link href="style03.css" rel="stylesheet">

<script src="jquery-1.11.1.min.js"></script>

$<$ link rel="stylesheet" href="nivo-slider/themes/default/default.css"/>

<link rel="stylesheet" href="nivo-slider/nivo-slider.css"/>

<script type="text/javascript" src="nivo-slider/jquery.nivo.slider.js"></ script>

<script src="js/highcharts.js" $></$ script $>$

<script src="js/modules/exporting.js" $></$ script $>$

$<$ style type="text/css" $>\$\{$ demo.css $\}</$ style $>$

<script type="text/javascript">

$\$($ function ()$\{$ var datos $=\$\{$ json $\}$

$\$(' \#$ estadistica1').highcharts $(\{$

chart: \{plotBackgroundColor: null, plotBorderWidth: 1, plotShadow: false\},

title: \{text: 'Resultados porcentuales del Clustering del Petitorio'\},

tooltip: \{pointFormat: '\{series.name\}: <b>\{point.percentage:. $1 \mathrm{f}\} \%</ \mathrm{b}>$ '\},

plotOptions: \{ pie: \{allowPointSelect: true, cursor: 'pointer', dataLabels:

\{enabled: true, format: ' $<\mathrm{b}>\{$ point.name $\}</ b>$ : \{point.percentage:. $1 \mathrm{f}\} \%$ ',
29. style: $\{$ color: (Highcharts.theme \&\& Highcharts.theme.contrastTextColor) \| 'black'\}\}\}\},

30. series: [\{type: 'pie', name: ‘\% del Cluster', data: datos\}]\});\});

31. $</$ script $></$ head $><$ body $>$

32. <div id="topContent" $><$ div id="estadistica1" $><$ div $>$

33. $<\mathrm{h} 1 \mathrm{id}=$ "logo" $><$ a href="\#" $>$ Prototipo Aplicación de Minería de Datos $<|\mathrm{a}\rangle<1$ h1 $1<<$ div $><!--$ topContent --><div id="container">

34. <div id="cabecera" $><$ form action="CTRL_Petitorio" method="POST">

35. <input type="hidden" name="nombreJSP" value="/IU Petitorio.jsp" $\mid>$

36. <table class $=$ "sedes" $><$ tr $><$ th $><h 4>$ Distritos Judiciales: $</$ h $4></$ th $>$

37. $</$ tr $><$ tr $><t d><$ input type="checkbox" name="CSJ01"

value $=" 1$ " checked $>$ Corte Suprema $<$ br $></ \mathrm{td}\rangle<\mathrm{td}>$

$<$ input type $=$ "checkbox" name="CSJ02" value $=" 2$ " $>$ Corte Lima Norte $<$ br $><$ < td $><$ tr $><$ tr $><$ td $><$ input type="checkbox" name="CSJ03" value="3" $>$ Corte Lima Sur $<$ br $>$

38. $</$ td $><$ td $><$ input type $=$ "checkbox" name="CSJ04" value $=" 4$ " $>$ Corte Lima Este $<$ br $></$ td $><\mid$ tr $><$ tr $><t d>N^{\circ}$ de Clusters: $<$ input type $=$ "number" name="ncluster" value="5" min="2" max="150" step="1" size="2" $><$ br $></$ td $><<$ tr $></$ table $><$ div id ="tarea" $><$ td $>\&$ \&bsp $:<$ tdd $>$ $<$ input type="submit" class="button_1" name="procesar" value="Procesar" $><$ input type="reset" class="button_1" name="reset value="Limpiar" $></$ div $></$ form $>$

$<$ div $><$ !-- cabecera -- $><$ div id="detalle" $><$ h4 $></$ h4 $><\%$ if $\left(m\right.$ resultado $!=$ null \&\& $m \_$resultado.getMiResult $($) size() $>0$ ) \{out.println(" $<\mathrm{h} 4><\mathrm{b}>$ Resultados de la Evaluación: $</ \mathrm{b}><1$ h4>");out.println(" $<$ td $><b>$ Error Cuadrático: "+String.valueOf(m_resultado. getErrorCuadrado())+" $</$ b $></$ td $>") ;\} \%>$

$42 . \quad<$ table class="tProductos" $>$

43. <\%if ( $m \_$resultado != null \&\& $\mathrm{m} \_$resultado.getMiResult().size ()$\left.>0\right)$ \{ out.print (“ $<\mathrm{td}><\mathrm{b}>\mathrm{N}^{\circ}$ Cluster $</ \mathrm{b}></$ td $>$ "); out.print( $<$ td $><\mathrm{b}>$ Instancias $</ \mathrm{b}></ \mathrm{td}>$ "); out.print (“<td $><b>$ Instancias $(\%)</$ b $></$ td $>$ "); out.print ( $<\langle\mathrm{td}><\mathrm{b}>$ Caracteristicas del Cluster $</ \mathrm{b}></$ tdd $>$ ")

for(int $i=0 ; i<m \_$resultado.getMiResult().size(); $\left.i++\right)\{$ out.println(“<tr>");

result=String.valueOf(m resultado.getMiResult().get(i).getUid() + 1): out.println(" $<$ td $>$ " result + " $</$ td $>$ ");

result=String.valueOf(m_resultado.getMiResult().get(i).getInstancias ()$)$; out.println("<td>" + result + "</td>");

result=String.valueOf(Math.round( $m$ resultado.getMiResult().get(i). getPorcentaje( ()$\left.\left.^{\star} 100.0\right) / 100.0\right)$;out println (“<td $>$ " + result + “</td > );

result=String.valueOf(m_resultado.getMiResult().get(i).getDetalle()); out.println( $<<$ td $>$ " + result + "</td $>$ ");

\section{rs . Prototipo Aplicación de Minería de Datos}

\footnotetext{
Distritos Judiciales:

$\square$ Corte Suprema

$\square$ Corte Lima Sur

N.6. $\mathrm{N}^{\circ}$ de Clusters: 5

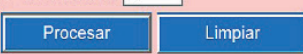

$\square$ Corte Lima Norte

$\square$ Corte Lima Este
}



QResultados de la Evaluación:

Error Cuadrático: 24831.0

$\mathbf{N}^{\circ}$ Instancias Instancias Caracteristicas del Cluster

$4384 \quad 42.8 \quad 2009$, Casacion

$1910 \quad 18.6$

$1691 \quad 16.51$ ESPECIAL

2009,Casacion,Previsional,Abreviado,'Impugnación Judicial de Acto Administrativo','Ley - 23908', 'PENSION DE JUBILACION' 2010,Casacion,'Contencioso Administrativo',Abreviado,'Acción Contencioso Administrativa','Ley - 24041','PAGO DE

NTERESES LEGALES'

2010, Casacion 'Contencioso Administrativo', Fspecial 'Acción Contencioso Administrativa', 'Ley - 24041','NULIDAD DE

RESOLUCION ADMINISTRATIVA'

$7827.63 \quad 2009$, Casacion,'Contencioso Administrativo',Abreviado,NINGUNO,'D. Leg - 276','NULIDAD DE RESOLUCION

ADMINISTRATIVA'

Figura 3: Prototipo de la Aplicación

Fuente: Elaboración propia 


\section{RESULTADOS Y DISCUSIÓN}

Los resultados obtenidos nos permitirán fundamentar los objetivos del presente trabajo. La muestra poblacional de análisis corresponde a un órgano jurisdiccional casatorio del periodo 2008 al 2010, la figura 4 grafica la distribución porcentual de los resultados obtenidos los cuales se analizan a continuación:

La interpretación de los conglomerados resultantes nos llevan a determinar que el $42.8 \%$ de la carga procesal corresponden a procesos casatorios iniciados en el año 2009 correspondiente a la subespecialidad contencioso administrativo en la cual la parte demandante solicita una bonificación especial fundamentando su pedido en el Decreto de Urgencia D.U 037-94.

El segundo conglomerado explica que se tiene un $18.65 \%$ de casos iniciados en el año 2009 solicitan Pensión de Jubilación y se fundamentan en la ley 23908 del código procesal civil; este pedido lo realizan mediante una impugnación judicial de acto administrativo.

\section{DISTRIBUCION DE INSTANCIAS POR N ${ }^{\circ}$ CLUSTER}

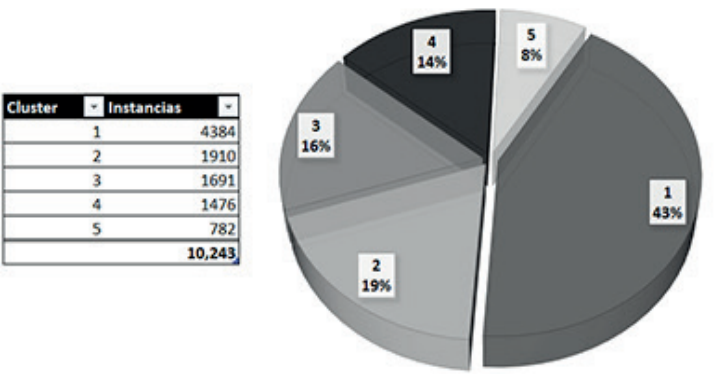

Figura 4: Distribución porcentual de la Evaluación

Fuente: Elaboración propia

El tercer conglomerado que equivale al $16.51 \%$ de la carga evaluada se trata de un pedido de Pago de Intereses Legales sosteniendo su pedido en la ley 24041, esta formulación se realiza mediante un proceso Abreviado con acción contencioso administrativo.

El cuarto conglomerado recae en un $14.41 \%$ quienes solicitan Nulidad de Resolución Administrativa respaldando su pedido mediante ley 24041 , este petitorio lo formulan mediante un proceso especial de acto administrativo.

El quinto conglomerado presenta el porcentaje más bajo, el $7.63 \%$ de la carga procesal tiene como requerimiento una Nulidad de Resolución Administrativa y lo realizan mediante un proceso abreviado de tipo acción contencioso administrativa, se fundamenta el petitorio en el Decreto Ley 276 del Código Civil.

\section{CONCLUSIONES}

Se ha propuesto un algoritmo de clustering distribuido adaptable a la entidad judicial por su naturaleza organizacional: esquemas de negocios dispersos físicamente, confidencialidad de la información, reúso de infraestructura tecnológica, complejidad organizacional, bajo presupuesto.

Se diseñó un modelo multidimensional que ha posibilitado organizar la información experimental basada en data nominal del proceso petitorio.

Se desarrolló un prototipo de software el cual ha permitido aplicar el algoritmo propuesto, el modelo multidimensional planteado y el uso de tecnologías Ad hoc a fin de predecir patrones de comportamiento en el petitorio de las partes procesales y exponer los resultados de la presente investigación.

La implementación de la presente propuesta en la organización del presente caso de estudio apoyará en el cumplimiento de los objetivos señalados en la agenda estratégica institucional como en el fortalecimiento de mecanismos para la reducción del volumen procesal, plazos procesales y nivel de litigiosidad; con lo cual se logrará una mejora en la calidad de los servicios que esta brinda a los ciudadanos.

\section{REFERENCIAS BIBLIOGRÁFICAS}

[1] Breña, W. (2008). La carga procesal bajo la lupa por materia y tipo de organo jurisdiccional. Justicia Viva, Volumen (1)

[2] Hurtado, F. (2005). Segmentación de clientes usando el algoritmo de clustering K-Mean. (tesina de pregrado). UNMSM, Lima

[3] Kimball, R., Reeves, L., Ross, M. y Thornthwaite, W. (2002). The Data Warehouse Lifecycle Toolkit. USA: Wiley

[4] Mendoza, E. (2013). Agenda Estrategica del Poder Judicial. Recuperado de https://www. pj.gob.pe

[5] PMSJ, (2014). Proyecto de Mejoramiento de los Servicios de Justicia. Recuperado de http://www.pmsj.org.pe

[6] Pressman, R. (7 Ed.) (2010). Ingenieria de Software Un enfoque Practico. (pp. 215-233) Mexico: Mc Graw Hill

[7] Rekha, S. y Sabu, M. (2010). Survey on Distributed Data Mining in P2P Networks. Recuperado de https://arxiv.org

[8] SGPPCM (2013). Política Nacional de Modernización de la Gestión Pública al 2021. Recuperado de http://www.pcm.gob.pe

[9] Veramendi, E. (2008). El petitorio implícito en los procesos de familia: A propósito del tercer pleno casatorio. Recuperado de http://boletinderecho.upsjb.edu.pe 